\title{
Designing Smart Multipurpose Digital Clock using Real Time Clock (RTC) and PIC Microcontroller
}

\author{
Sadeque Reza Khan \\ Lecturer, Dept. of EEE \\ Prime University \\ Dhaka, Bangladesh
}

\author{
Alvir Kabir \\ Dept. of ETE \\ University of Liberal Arts \\ Bangladesh
}

\author{
Dilshad Ara Hossain \\ Dept. of Physics \\ Eden Mohila College, Dhaka
}

\begin{abstract}
Now-a-days people prefer digital clocks more than an analog clock because of their elegant outlook, inexpensiveness, small size and accuracy. As a result many smart digital clocks are manufactured by different popular companies. Because of its low price and tiny size, it is often incorporated into all kinds of devices such as cars, radios, televisions, microwave ovens, standard ovens, computers and cell phones to enhance the quality of that device. In this particular project, design of a smart multipurpose clock is provided. This clock is designed with two PIC microcontrollers and a Real Time Clock (RTC) IC. The display section is designed with Seven Segment display and LED. A temperature sensor is also integrated with the system to show the current temperature in Seven Segment.
\end{abstract}

\section{General Terms}

Real Time Clock, Temperature, Time, Date, Seven Segment, Microcontroller.

\section{Keywords}

$\mathrm{I}^{2} \mathrm{C}$ communication, LED, 16F877A, LM35DZ.

\section{INTRODUCTION}

A digital clock is no different than an analog clock. It simply handles the timing functions electronically rather than mechanically [1]. A digital clock is a type of clock that displays the time digitally, i.e. in the LCD, LED or Seven Segment display, as opposed to an analog clock, where the time is displayed by hands [2]. Electronic clocks have predominately replaced the mechanical clocks as they are much reliable, accurate, maintenance free, portable and cost effective [3]. In this project work a multipurpose digital clock is developed which not only shows the time operations but also indicates the room temperature. The whole controlling operation is maintained by a PIC microcontroller 16F877A which not only communicates with a Real Time Clock IC but also calculates the whole time functions and displays it with Seven Segment and LED displays. Another PIC microcontroller 16F72 is used to enhance the number of ports and synchronizes with main controller IC to display the days in the LED. A real-time clock (RTC) is used to keep track of the current time. Although the term often refers to the devices in personal computers, servers and embedded systems, RTCs are present in almost any electronic device which needs to keep accurate time [4] for precise work to be maintained.

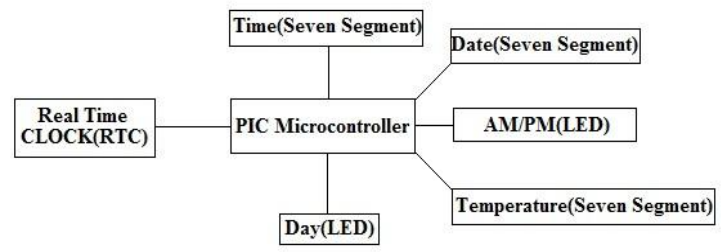

Fig 1: Overall proposed system

\section{OVERALL SYSTEM}

An overall block representation of the proposed smart multipurpose clock is shown in figure 1 . In this system a main controller IC PIC 16F877A is used to synchronize the operation of a RTC IC DS1307 and Seven Segments. The time and date are displayed by Seven Segments whether the day and AM/PM operations are displayed with LED. There is an additional option in this clock. It also displays the temperature in Seven Segments.

\section{HARDWARE DESIGN}

\subsection{Main Controller Section}

The control module is built with the microcontroller IC. The central controller is Microchip PIC16F877A. PIC 16F877A is an upper range and 16 series low cost 8 bit microcontroller [5], [6]. It consists of $33 \mathrm{I} / \mathrm{O}$ (Bi directional lines) with $25 \mathrm{~mA}$ current in per pin. It also has 5 channel built-in A/D converter and serial communication. Its synchronous serial port can be configured as either 3-wire Serial Peripheral Interface (SPI) or the 2-wire Inter-Integrated Circuit $\left(\mathrm{I}^{2} \mathrm{C}\right)$ bus. Universal Asynchronous Receiver Transmitter (UART) is another useful feature of this device. Another PIC microcontroller $16 \mathrm{~F} 72$ is used in this project to show the DAY option of the digital clock. It is synchronized with 16F877A. PIC $16 \mathrm{~F} 72$ is a 28 pin 8 -bit microcontroller with 8-bit ADC [7].

\subsection{Real Time Clock DS1307}

Clocked by $32.768 \mathrm{kHz}$ crystal; the Maxim DS1307 is one of the popular I² 8 -pins RTC chip available on the market [8], [9]. Equipped with Automatic Power-Fail Detect and Switch 
Circuitry the Maxim DS1307 will continue to operate accurately even though the main power supply is lost and because it's consumed less than 500nA on 3 volt lithium backup battery (48mAhr or greater), the DS1307 will continue to operate more 10 year in the absence of the main power supply. The DS1307 is capable to count accurately the second, minute, hour, day of the week, date of the month, month and year include the leap year until the year 2100; with its $\mathrm{I}^{2} \mathrm{C}$ interface capabilities make this chip easily to be integrated with widely available microcontrollers that have build in $\mathrm{I}^{2} \mathrm{C}$ peripheral.

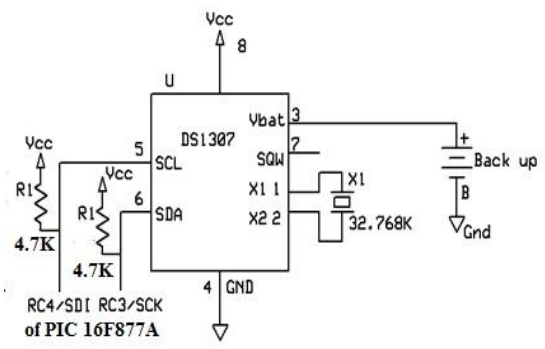

Fig 2: Connection Diagram of DS1307

\subsubsection{I2C Protocol}

$\mathrm{I}^{2} \mathrm{C}$ is a serial data bus protocol that allows multiple devices to connect to each other with fairly slow data transfer rates for better collaboration [10]. Many microcontrollers have libraries to support $\mathrm{I}^{2} \mathrm{C}$. The $\mathrm{I}^{2} \mathrm{C}$ bus use only 2 bidirectional data lines for communicating with the microcontroller. The $\mathrm{I}^{2} \mathrm{C}$ protocol use master and slave method, the master which is usually the microcontroller while the slave can be any $\mathrm{I}^{2} \mathrm{C}$ device such as Real Time Clock DS1307. $\mathrm{I}^{2} \mathrm{C}$ communication requires two ports, one for the serial data called SDA (serial data) to communicate with SCK pin of PIC IC and the other for synchronize clock called SCL (serial clock) to communicate with SDI pin of PIC IC[11].

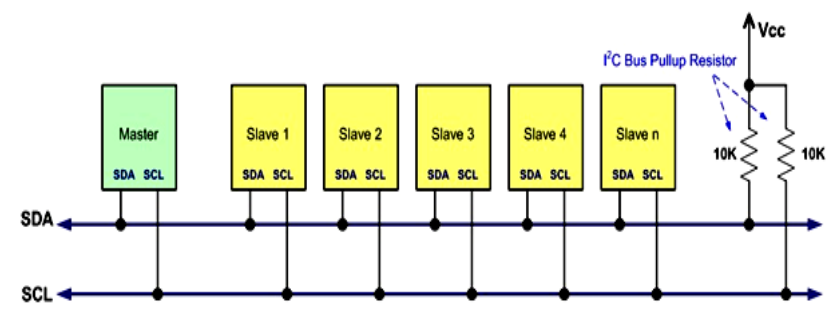

Fig 3: Typical I2C device connection

\subsection{Seven Segment Display}

A seven-segment display (SSD), or seven-segment indicator, is a form of electronic display device for displaying decimal numerals that is an alternative to the more complex dot-matrix displays. Seven-segment displays are widely used in digital clocks, electronic meters, and other electronic devices for displaying numerical information. The segments themselves are identified with lower-case letters "a" through "g," with segment "a" at the top and then counting clockwise. Segment "g" is the center bar. Most seven-segment digits also include a decimal point ("dp") [12]. There are two types of displays available, common anode and common cathode [13]. In this project we use common cathode Seven Segment display to show the time, date and temperature option.

\subsection{Sensor Section}

A temperature sensor is used in this project to display the room temperature. For measuring room temperature LM35DZ is used. The LM35DZ is a precission semiconductor temperature sensor giving an output of $10 \mathrm{mV}$ per degree Centograde [14].
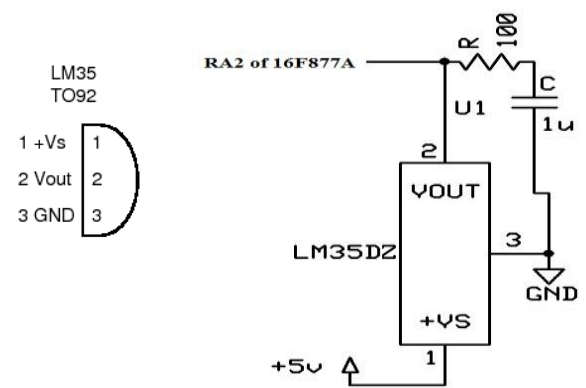

Fig 4: (a) LM35DZ, (b) Connection Diagram of LM35DZ

\section{WORKING PRINCIPLE}

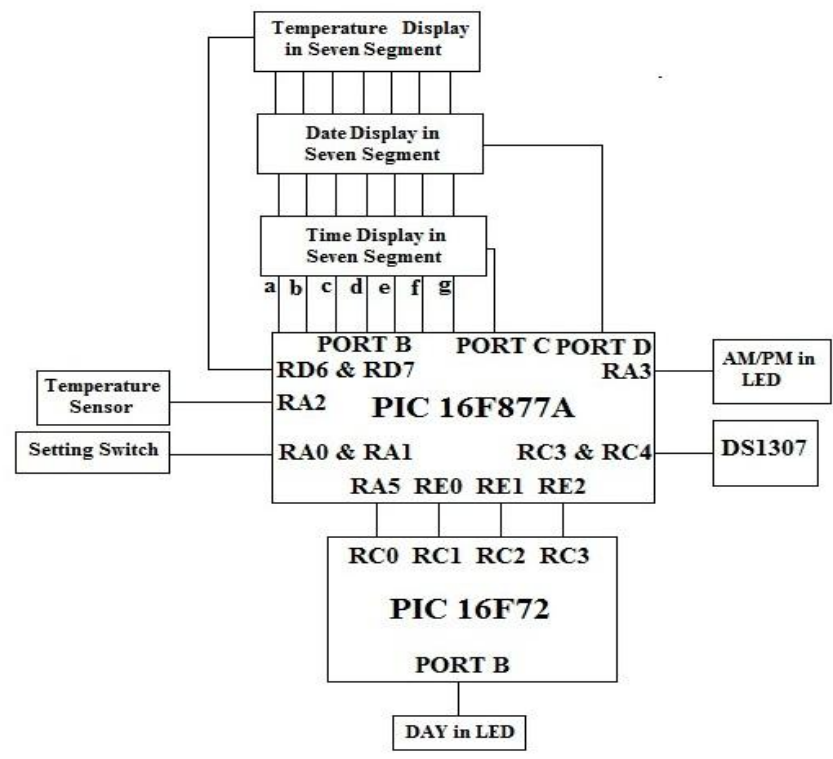

Fig 5: Connection Diagram of Multipurpose Digital Clock

In this project DS1307 IC operates as a slave and it communicates with the main controller PIC 16F877A by $\mathrm{I}^{2} \mathrm{C}$ protocol. As a master PIC 16F877A can read and write data in DS1307 by using SDA and SCL ports. The whole clock operations are maintained by RTC DS1307. PIC 16F877A only reads the data from RTC DS1307 and shows it in common cathode Seven Segment displays. Six Seven Segments are used 
to show the time, six are used to show date and two are used to show temperature. The ports of Seven Segment, i.e. a, b, c, d, e, $\mathrm{f}$ and $\mathrm{g}$ pins are scanned by PORT B of main controller. To show the TIME, Port $\mathrm{C}$ of $16 \mathrm{~F} 877 \mathrm{~A}$ controls the cathodes of corresponding Seven Segments by switching of transistors. Again DATE and Temperature Seven Segments are controlled by PORT D of 16F877A. The temperature is measured by LM35DZ sensor and the data of the sensor is collected by ADC port RA2 of 16F877A. AM and PM are showed by two LEDs which are switched by a transistor from RA3 pin of main controller IC. For setting operation two touch switches (SET and UP) are used and those are connected in RA0 and RA1 pin of 16F877A. To connect Seven Segments, LEDs, switches, sensor and DS1307 IC, $29 \mathrm{I} / \mathrm{O}$ pins of 16F877A are used and only $4 \mathrm{I} / \mathrm{O}$ pins are left to show the DAY option of the designed clock. So another PIC 16F72 which is a low cost microcontroller is used to show the DAY option in seven LEDs. To maintain the current DAY a combination is provided to PIC $16 \mathrm{~F} 72$ by using the four pins of main controller.

\section{FLOW CHART}

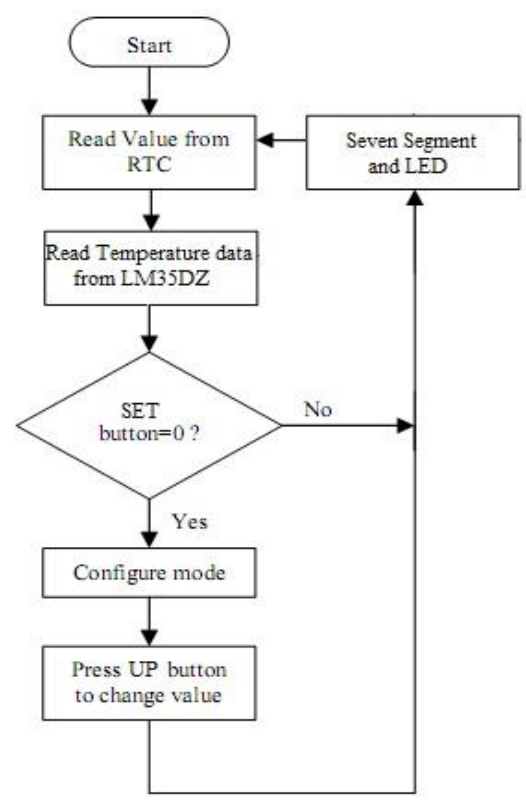

Fig 6: Main Macro of Overall Logic

The provided flow chart is the main macro of the overall program. It shows that the clock information's are read from RTC and the temperature data is read from the sensor and displayed. If the SET button is pressed then the system will enter in setting option and the initial data can be changed by using UP button.

\section{RESULT}

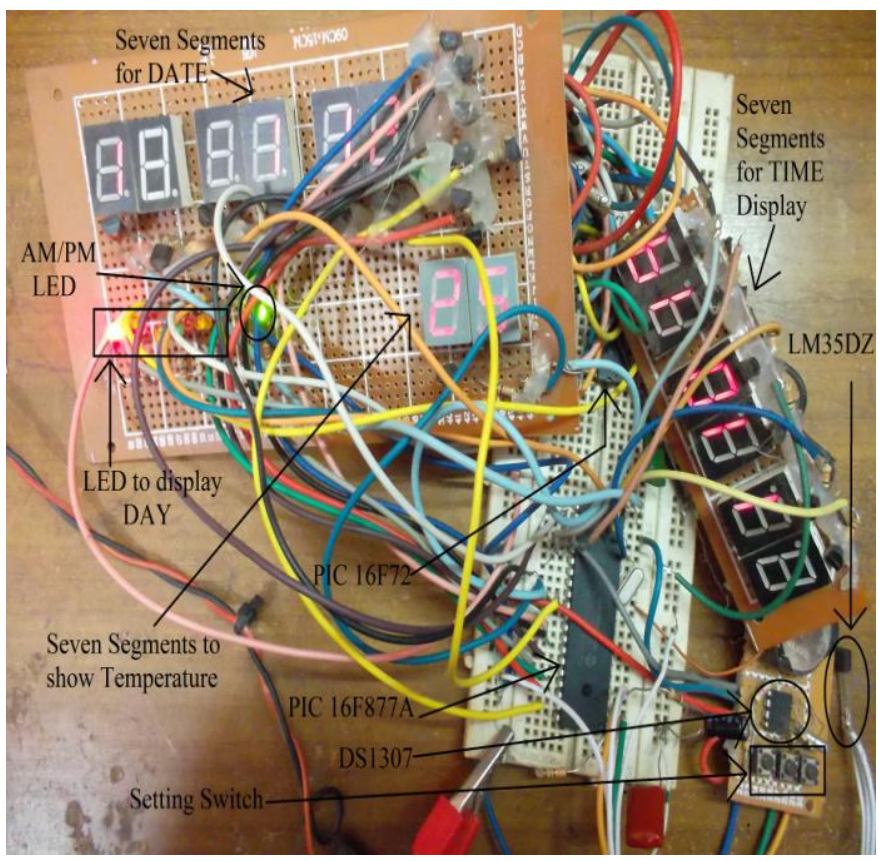

\section{CONCLUSION}

Now-a-days different pattern of digital clocks are available in market. Most of them are of very high price and low quality. Many of those cannot provide the time accurately for longer period as those are designed with timer IC's like 555 timer. Again some digital clocks loss their data whenever the power supply shuts down. But our designed multipurpose digital clock is accurate because of its Real Time Clock module that keeps track of the system time and update. The DS1307 has a built-in power sense circuit that detects power failures and automatically switches to the $3 \mathrm{~V} \mathrm{Li} \mathrm{Cell} \mathrm{battery} \mathrm{supply} \mathrm{which} \mathrm{is} \mathrm{incorporated}$ with the RTC. Most of the digital clock in the market does not cover all the time functions whether our designed digital clock covers all the time options and we will also incorporate the alarm option in next version. However, the Temperature displaying is an additional feature of our smart digital clock. It is possible to develop this system with only USD 7. So this version of digital clock is really a cheap, precise and well featured device for the present market.

\section{REFERENCES}

[1] How digital clock works (online). Available: http://electronics.howstuffworks.com/gadgets/clockswatches/digital-clock1.htm

[2] Digital Clock, 12 January 2012 (online). Available: http://en.wikipedia.org/wiki/Digital_clock

[3] Pan Thu Tun, "Development and Implementation of Microcontroller-based Digital Clock", World Academy of Science Engineering and Technology 42 2008, pp: 362365.

[4] Real Time Clock, 29 January 2012 (online). Available: http://en.wikipedia.org/wiki/Real-time_clock

[5] PIC16F87XA Data Sheet: 28/40/44-Pin Enhanced Flash Microcontrollers, (C) 2003, Microchip Technology Incorporated. 
[6] PIC Tutorial Hardware- PIC Vietnam (Online). Available: http://www.picvietnam.com/download /Nigel\%tutorial.pdf

[7] PIC 16F72, 31 January 2012 Available: http://ww1.microchip.com/downloads/en/devicedoc/39597 b.pdf

[8] Booting $\mathrm{CD} / \mathrm{M} 3$ from an SD card, Available: http://benryves.com/journal/tags/DS1307

[9] DS1307 RTC Real time clock (online). Available:http://www.ladyada.net/learn/breakoutplus/ds130 7rtc.html

[10] Display Real Time Clock (RTC) On LCD, 5 February 2012.Available:http://www.cytron.com.my/attachment/Det ails\%20Description/PR12\%20v4.pdf
[11] Sagar. G. Yadav, K. A. Narayanankutty, "A Versatile Industrial Timer and Real Time Keeper", Wireless Engineering and Technology, 2011, 2, 196-203.

[12] The Seven Segment LED, 12 January, 2012 (online). Available: http://www.engineersgarage.com/electroniccomponents/7-segment-display

[13] Lecture 7. Digital Display, 20 January, 2012. Available:http://hibp.ecse.rpi.edu/ connor/education/IEE/le ctures/Lecture_7_digital_display.pdf

[14] LM35DZ Available: http://www.alldatasheet.net/ datasheet -pdf/pdf/8875/NSC/ LM35DZ.html 